\title{
Combined Catalysis and Optical Screening for High Throughput Discovery of Solar Fuels Catalysts
}

\author{
J. M. Gregoire, ${ }^{\mathrm{a}, \mathrm{z}}$ C. Xiang, ${ }^{\mathrm{a}}$ S. Mitrovic, ${ }^{\mathrm{a}}$ X. Liu, ${ }^{\mathrm{a}}$ M. Marcin, ${ }^{\mathrm{a}}$ E. W. Cornell, ${ }^{\mathrm{b}}$ J. Fan, \\ and J. Jin ${ }^{\mathrm{a}, \mathrm{b}, *}$ \\ a Joint Center for Artificial Photosynthesis, California Institute of Technology, Pasadena, California 91125, USA \\ ${ }^{b}$ Engineering Division, Lawrence Berkeley National Laboratory, Berkeley, California 94720, USA \\ ${ }^{c}$ Department of Chemistry, Zhejiang University, Hangzhou, Zhejiang Province 310027, China
}

Considerable research and development efforts are being devoted to the efficient generation of solar fuels. A solar fuels device couples a solar photoabsorber with catalysts to convert solar energy to chemical energy via reactions such as oxygen evolution (water splitting). Widespread deployment of this technology hinges upon discovery of new materials through efforts such as the high throughput screening of oxygen evolution catalysts, as discussed in this manuscript. We derive an expression for the efficiency of the oxygen evolution catalyst that combines catalytic and optical properties. Using this hybrid efficiency, we screen 5456 samples in a $(\mathrm{Fe}-\mathrm{Co}-\mathrm{Ni}-\mathrm{Ti}) \mathrm{O}_{\mathrm{x}}$ pseudo-quaternary catalyst library using automated, high throughput electrochemical and optical experiments. The observed compositional trends in this catalyst efficiency lead to the discovery of a new high performance composition region. (C) 2013 The Electrochemical Society. [DOI: 10.1149/2.035304jes] All rights reserved.

Manuscript submitted December 10, 2012; revised manuscript received January 21, 2013. Published January 30, 2013. This was Paper 1716 presented at the Honolulu, Hawaii, Meeting of the Society, October 7-12, 2012.

Solar fuel technology offers the formidable capability to convert solar energy and store it in chemical bonds. Given the easy storage and transport of liquid fuel, solar fuel generation is an attractive technology for the renewable energy landscape. ${ }^{1,2}$ The widespread deployment of this technology requires a device fabricated with high-performance, robust materials made from earth-abundant elements. ${ }^{3}$ The realization of this device relies on the discovery of new materials, prompting an aggressive high-throughput materials discovery effort within the Joint Center for Artificial Photosynthesis (JCAP, http://solarfuelshub.org/). This effort includes searches for new photoanodes, photocathodes, and catalysts for several solar fuels reactions, of which the oxygen evolution catalyst is discussed in this manuscript.

One established solar fuel generator concept combines a solar photoabsorber with an optically inactive heterogeneous catalyst to evolve oxygen (water splitting). "That is, the oxygen evolution reaction (OER) is mediated by a "dark" catalyst that is coupled to a photoabsorber, which provides the required potential and current for the electrocatalytic reaction through the photovoltaic effect. This anodic reaction balances a fuel-producing cathodic reaction such as hydrogen evolution or $\mathrm{CO}_{2}$ reduction. Variations within this solar fuel design concept include the use of a tandem absorber containing a coupled photoanode and photocathode instead of a single photoabsorber ${ }^{5-7}$ or the use of a homogeneous OER catalyst instead of the heterogeneous catalyst. ${ }^{3,8-11}$ Homogeneous catalysts are not considered in this manuscript, and the general discussion of optimizing the OER catalyst is applicable to any photoabsorber device. Our primary assumptions concerning device architecture are that the OER catalyst coats the photoanode and this anode is exposed to solar illumination. These assumptions are based upon a tandem photoabsorber system in which the photoanode is the top cell and has a larger bandgap than the photocathode.

While heterogeneous catalysts are used in a variety of technologies, the OER catalyst in this solar application has a special optical performance requirement. Since the solar radiation impinges the catalyst before reaching the photoabsorber, the catalyst layer must be sufficiently transparent. Since the optical properties of the electrocatalyst could play an important role in the overall solar-to-fuel conversion efficiency, the evaluation of these properties should be incorporated into a high-throughput search for new solar fuels OER catalysts.

While the ultimate development of a solar fuels device requires careful integration of the device components, high-throughput discovery and optimization is most practically performed on each component individually. ${ }^{3,4,12-14}$ Such studies require a heuristic figure of

*Electrochemical Society Active Member.

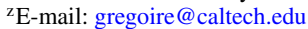

merit to evaluate the performance of the independent material. We derive such a figure of merit (FOM) for the oxygen evolution catalyst by combining the electrocatalytic performance and optical transparency under solar air mass 1.5 irradiance. The resulting FOM for the overall catalyst efficiency is evaluated for 5456 (Fe-Co-Ni-Ti) $\mathrm{O}_{x}$ catalysts using automated, high-throughput electrochemical and optical experiments. These catalysts cover the quaternary metal space with 3.33 at.\% interval and the optimal composition region for the overall catalyst efficiency is markedly different than the optimal composition region for electrocatalytic activity.

The combination of techniques and application of the efficiency model described in this manuscript comprise a new high-throughput technique for evaluating OER catalysts. Previous combinatorial studies of OER electrocatalysis and photoelectrocatalysis systems ${ }^{12-14}$ do not provide the necessary information to identify an optimal catalyst for the device architecture described above. The electrochemical and optical measurements employed in this study provide archived data of catalytic current as a function of overpotential and transmission as a function of wavelength, which may be used to assess a catalyst's performance for any photoabsorber system. This capability will be quite useful since new photoabsorbers are being discovered and developed in parallel with the catalyst research. This tailoring of material characterization techniques for solar fuels applications and the deployment of high-throughput realizations of these techniques will streamline the catalyst discovery process by ensuring that the high-throughput screening criteria are directly applicable to operational devices.

\section{Figures of Merit for Solar Fuel OER Catalysts}

Exploration of composition trends in multi-dimensional parameter space, such as quaternary composition space, is most effectively performed by mapping a scalar figure of merit over the parameter space. For the purposes of screening OER catalysts for solar fuels applications, we describe figures of merit for catalytic activity, solar transparency and overall catalyst efficiency.

Catalytic activity figure of merit.- The performance of an OER electrocatalyst is most explicitly characterized by the molar oxygen production rate at a given overpotential, $V_{O E R}$. In electrochemical characterization, one instead measures the anodic current density $J_{\mathrm{C}}\left(V_{O E R}\right)$ as a function of the overpotential and carries out additional experiments to access the Faradic efficiency for $\mathrm{O}_{2}$ production. A figure of merit for the electrochemical characterization can be defined as either the minimum $V_{O E R}$ required to obtain a desired current density, or as the maximum $J_{\mathrm{C}}$ attained at a given overpotential. 
Using the latter definition, we employ high throughput methods to map $J_{\mathrm{C}}(450 \mathrm{mV})$ across our catalyst library.

Optical transparency figure of merit.- During incorporation of the OER catalyst and the photoabsorber, the catalyst transmittance must be considered for proper light management and optimal use of the incident solar power. Using the ASTM 6173-03 standard for global tilt air mass 1.5 (AM1.5) spectral irradiance, $\boldsymbol{E}_{\mathrm{AM} 1.5}$, the electrical power density generated by the photoabsorber system is given by

$$
\boldsymbol{P}=\int E Q E_{\mathrm{PA}}(\lambda) T_{\mathrm{C}}(\lambda) \boldsymbol{E}_{\mathrm{AM} 1.5}(\lambda) \mathrm{d} \lambda,
$$

where $E Q E_{\mathrm{PA}}$ is the spectral external quantum yield of the photoabsorber and $T_{\mathrm{C}}$ is the spectral transmittance of the catalyst. In the development of a catalyst for a given photoabsorber, catalyst optimization can be performed using the known $E Q E_{\mathrm{PA}}$ spectrum. In the development of OER catalysts for a generic photoabsorber, a corresponding figure of merit is the transmission efficiency of the solar irradiance over a wavelength range of interest:

$$
\eta_{\mathrm{C}, T}=\int_{\lambda_{1}}^{\lambda_{2}} T_{\mathrm{C}}(\lambda) \boldsymbol{E}_{\mathrm{AM} 1.5}(\lambda) \mathrm{d} \lambda / \int_{\lambda_{1}}^{\lambda_{2}} \boldsymbol{E}_{\mathrm{AM} 1.5}(\lambda) \mathrm{d} \lambda .
$$

This normalized efficiency approaches zero for opaque catalysts and unity for transparent catalysts. The numerator integral is essentially the same as Eq. 1 with $E Q E_{\mathrm{PA}}$ equal to 1 between $\lambda_{1}$ and $\lambda_{2}$ and 0 elsewhere. A practical value for the high wavelength limit $\lambda_{2}$ is that of the anticipated (lowest energy) bandgap of the photoabsorber. The low wavelength limit $\lambda_{1}$ can be chosen to be near or below 400 $\mathrm{nm}$ because the spectral irradiance is relatively small beyond this limit.

Combined figure of merit. - To combine catalytic activity and optical transparency performance, further knowledge of the photoabsorber and cathode catalyst system is required. A photoabsorber is most traditionally characterized by $J_{\mathrm{PA}}\left(V_{p h}\right)$ performance, the standard photovoltaic current-voltage relationship. For solar water splitting the current density $J_{\mathrm{PA}}$ must match the catalytic current for both the anodic and cathodic reactions, with respective overpotentials provided by the photovoltage $V_{p h}$.

The mathematical expression for the efficiency of a water splitting device is a recursive function of the performance metrics of the individual components. For the present purpose of high throughput electrocatalyst screening, we require an expression for catalyst efficiency that is independent of the detailed device physics of the absorber materials. This efficiency can be succinctly stated in terms of two photoabsorber parameters: $V_{\mathrm{PA}, O E R}$ and $J_{\mathrm{PA}, \max }$. At its operating photovoltage $V_{p h}$, the photoabsorber supplies an OER overpotential to the OER catalyst, which we define as $V_{\mathrm{PA}, O E R}$. During the photoabsorber's independent operation at this voltage, the photocurrent is given by the $J_{\mathrm{PA}}\left(V_{p h}\right)$ characteristic. Due to the current-matching constraint for the photoabsorber and catalyst, this is the maximum operating current of the integrated device and is defined as $J_{\mathrm{PA} \text {,max }}$. Since the catalyst needs to provide an OER catalytic current no greater than $J_{\mathrm{PA} \text {, max }}$, the catalytic efficiency is given by

$$
\eta_{\mathrm{C}, O E R}=\operatorname{Min}\left[J_{\mathrm{C}}\left(V_{\mathrm{PA}, O E R}\right), J_{\mathrm{PA}, \max }\right] / J_{\mathrm{PA}, \max } \cdot
$$

In essence, this expression gives the catalytic efficiency of $\mathrm{O}_{2}$ production compared to the maximum attainable with a given photoabsorber.

The photoabsorber will only be able to operate at this limit if the catalyst is perfectly transparent. Precise modeling of the device efficiency as a function of catalyst transparency requires knowledge of the irradiance-dependence of $J_{\mathrm{PA}}\left(V_{p h}\right)$. For the present purpose, we assume the device efficiency varies linearly with the irradiance transmittance of the catalyst (Eq. 2). Thus, the overall efficiency of the catalyst can thus be approximated by combining the catalytic and optical efficiencies:

$$
\eta_{\mathrm{C}}=\eta_{\mathrm{C}, T} \eta_{\mathrm{C}, O E R}
$$

Given the photoabsorber and wavelength parameters in Eqs. 2 and 3, this figure of merit can be used to evaluate and develop OER catalysts.

\section{Experimental}

Library synthesis.- An ink-jet printing assisted cooperativeassembly method (IJP-A) has been applied for the high-throughput generation of the $(\mathrm{Fe}-\mathrm{Co}-\mathrm{Ni}-\mathrm{Ti}) \mathrm{O}_{\mathrm{x}}$ pseudo-quaternary catalyst library with established metal precursor formulations. These formulations, as previously described by Liu et al. ${ }^{15}$ contained block copolymer structure directing agents, which have been developed in conjunction with a drying/precipitation and calcination protocol to yield porous metal oxide thin-film samples. Briefly, each Fe-Co-Ni-Ti composition in the library was synthesized by combining four "inks", each with an elemental precursor $\left(\mathrm{FeCl}_{3}, \mathrm{Co}\left(\mathrm{NO}_{3}\right)_{2} \bullet 6 \mathrm{H}_{2} \mathrm{O}, \mathrm{Ni}\left(\mathrm{NO}_{3}\right)_{2} \bullet 6 \mathrm{H}_{2} \mathrm{O}\right.$ and $\mathrm{TiCl}_{4}$ obtained from Sigma Aldrich). Using vigorous stirring, $5 \mathrm{mmol}$ of each metal precursor, $6 \mathrm{mmol}$ of acid $\left(\mathrm{HCl}\right.$ or $\left.\mathrm{HNO}_{3}\right)$ and $0.5 \mathrm{~g}$ of $\mathrm{P} 123\left(\mathrm{EO}_{20} \mathrm{PO}_{70} \mathrm{EO}_{20}, \mathrm{MW}=5800\right.$; Sigma Aldrich $)$ was dissolved into $45 \mathrm{~mL}$ ethanol. The ink formulation and physicochemical properties have been detailed in. ${ }^{15}$ Using the inkjet printer (modified Epson Stylus Pro 4880), each composition sample was deposited onto a $1 \mathrm{~mm}^{2}$ substrate area by dispensing a fixed volume of solution. The volumetric ratio of the inks was chosen to provide the desired metal stoichiometry, and each sample contained approximately $10 \mathrm{nmol}$ of metal.

The library included all possible compositions in the $\mathrm{Fe}-\mathrm{Co}-\mathrm{Ni}-\mathrm{Ti}$ composition space with 3.33 at.\% intervals, and we note that in this manuscript all referenced concentrations are with respect to the metal composition. The 5456 samples were each deposited in a $1 \mathrm{~mm}$ square footprint. A regular grid of samples with $2 \mathrm{~mm}$ pitch was evenly distributed onto 3 glass plates coated with $400 \mathrm{~nm}$ of fluorine-doped tin oxide (FTO) (TEC15 glass supplied by Hartford Glass Co., Hartford, IN; FTO sheet resistance 12-14 Ohms per square). The as-deposited precursor library was then calcined at $350^{\circ} \mathrm{C}$ for $5-6$ hours in a box furnace with flowing air. This calcination efficiently volatilized and removed the precursor ligands and other ink components, and additionally yielded oxidation of the metals. The resulting samples were porous, non-contiguous metal oxide coatings with nominal thickness of approximately $300 \mathrm{~nm}$. Each sample was well adhered to the FTO coating and was contained within the designed $1 \mathrm{~mm}$ square electrode area. The grid of samples on each plate shared the common FTO contact layer.

High throughput electrochemistry.- High throughput electrochemical experiments were performed on each library using our novel scanning droplet cell and a Gamry G 300 potentiostat controlled with custom automation software. The cell design, described in detail by Gregoire et al., ${ }^{16}$ provides the capability to perform standard 3-electrode electrochemical characterization of each sample in the catalyst library. The cell was outfit with a Pt wire counter electrode and custom made $\mathrm{Ag} / \mathrm{AgCl}$ reference electrode. A standard 2-axis translation stage was used to raster the library below the fixed droplet cell. The characterization of each sample included the establishment of a stable solution contact to the sample plate with $2.5 \mathrm{~mm}$ diameter, which coated a single $1 \mathrm{~mm}$ square sample. The gasket-free, continuous flow design of the scanning droplet cell provided flushing of the active cell volume every second so that fresh solution was provided to each sample.

To screen the library samples for OER electrocatalysis, a voltage sweep was performed from $-0.04 \mathrm{~V}$ to $0.52 \mathrm{~V}$ with respect to the $\mathrm{O}_{2} / \mathrm{H}_{2} \mathrm{O}$ Nernst potential in the aqueous electrolyte solution of $\mathrm{O}_{2}$ bubbled $1 \mathrm{M} \mathrm{NaOH}$. After approximately 0.8 seconds of translation to the sample, the working electrode was held at the starting potential of the $\mathrm{CV}$ for $2 \mathrm{~s}$. A settle time of $1 \mathrm{~s}$ was needed to stabilize the droplet volume and electrode contact, during which the working electrode was held at the starting potential of the voltage sweep to mitigate transients at the start of the experiment. At a scan rate of $0.15 \mathrm{~V} \mathrm{~s}^{-1}$, the voltage range was covered in $3.7 \mathrm{~s}$, which combined with the settle time and 


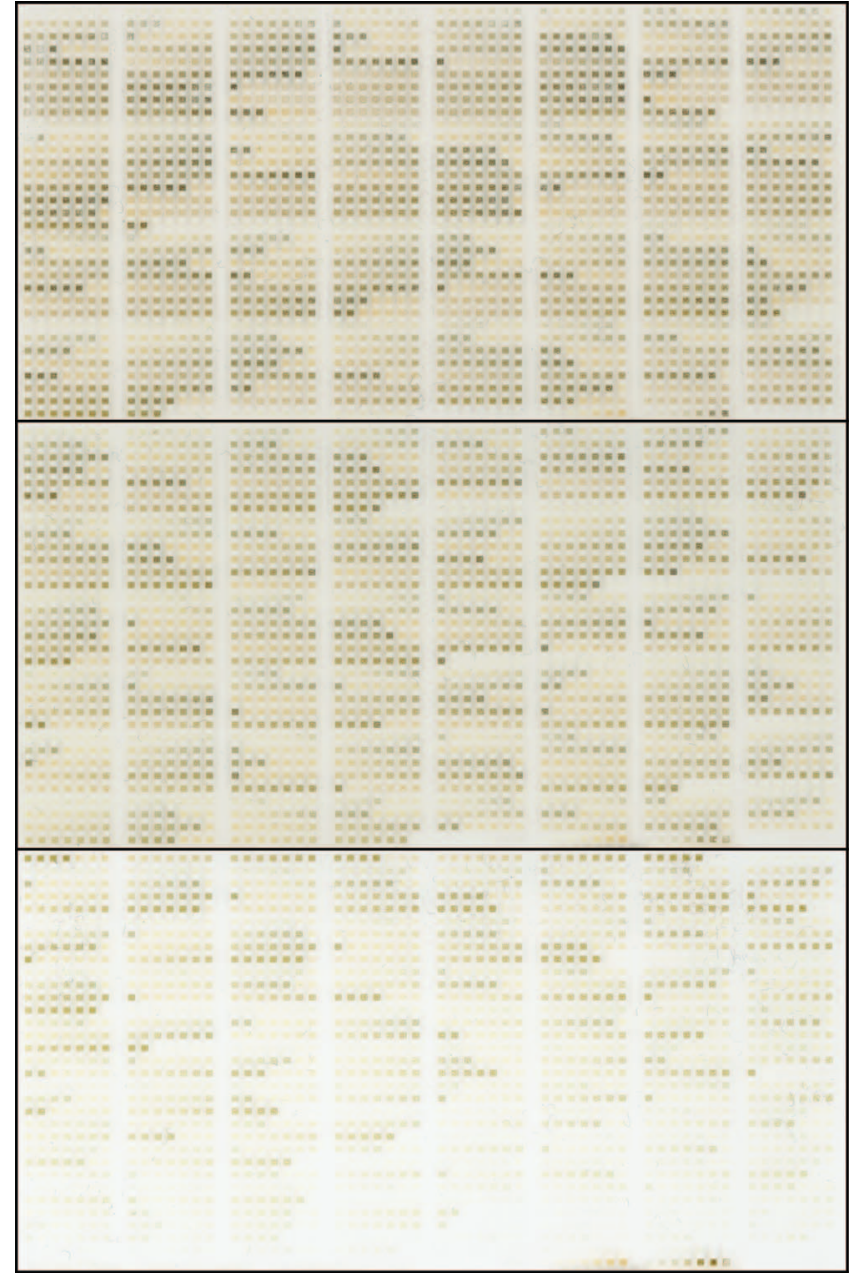

Figure 1. Images of the 3 plates containing the 5456 samples in the (Fe-Co$\mathrm{Ni}-\mathrm{Ti}) \mathrm{O}_{\mathrm{x}}$ library. The thin film samples are each $1 \mathrm{~mm}$ square and are arranged on a regular grid on the FTO coated glass plates.

$0.8 \mathrm{~s}$ translation between samples, yielded a sample throughput of 5.5 s per sample.

High throughput optical screening.- The optical transparency of each catalyst was measured after the electrochemical experiments using a fiber-coupled mercury arc lamp (Newport 66921, 450W-1000W) and an integrating sphere (Ocean Optics ISP-50-8-R-GT) coupled to a UV-visible spectrometer (Ocean Optics USB4000). The end of the $0.1 \mathrm{~mm}$ fiber was positioned $1.6 \mathrm{~mm}$ from the sample such that the divergent beam generated a circular footprint on the sample with diameter of approximately $1 \mathrm{~mm}$. Transmitted and low-angle scattered light was collected by the integrating sphere after transmission through the FTO coating, the $2.2 \mathrm{~mm}$ glass plate and a $2 \mathrm{~mm}$ air gap. The $8 \mathrm{~mm}$-diameter aperture of the integrating sphere and its $5.8 \mathrm{~mm}$ separation from the fiber termination provided a collection angle of $35^{\circ}$ with respect to the fiber and $44^{\circ}$ with respect to the sample.

The sample plate was mounted on a frame, which was cantilevered off a translation stage such that each sample could be positioned in the transmission measurement. Each transmission spectra was acquired with a $2 \mathrm{~s}$ integration time, which combined with $1 \mathrm{~s}$ translation time yielded a throughput of $3 \mathrm{~s}$ per sample. As shown in Fig. 1, the array samples were arranged on each plate such that every eighth column in the array did not contain samples. These bare positions were used as spectral reference sites, where a reference transmission measurement was made after every set of 7 measurements of catalyst samples. Using a computer-actuated shutter, dark spectra were also collected at these sites so that the a corrected, normalized spectral transmission was calculated for each sample as

$$
T_{\mathrm{C}}(\lambda)=\left(S_{\mathrm{c}}-S_{\mathrm{dark}}\right) /\left(S_{\text {ref }}-S_{\text {dark }}\right),
$$

where $S_{\mathrm{c}}$ and $S_{\text {ref }}$ are the measured spectra at the catalyst sample location and bare reference location, respectively, and $S_{\text {dark }}$ is the measured dark spectrum.

This spectral transmission was used in data interpretation, as described by Eq. 2, and the wavelength range of the transmission measurement constrained the integration range in this equation. While photoabsorbers with bandgap energy down to approximately $1 \mathrm{eV}$ are of interest for solar fuel generation, in this work we use a $\lambda_{2}$ value of $925 \mathrm{~nm}(1.35 \mathrm{eV})$. For $\lambda_{1}$ we use $400 \mathrm{~nm}(3.1 \mathrm{eV})$, below which lies less than $5 \%$ of the energy content of the AM1.5 spectrum. This wavelength range contains over $65 \%$ of the AM1.5 energy content and is a prime range of interest for solar photoanodes. ${ }^{3}$

\section{Results and Discussion}

Images of the 3 library plates acquired with a flatbed scanner (Epson V600 Photo) containing the 5456 samples are shown in Fig. 1. The 3-dimensional composition space is unfolded onto the 2-dimensional plates, with the only monotonic composition trend being increasing Ti content with lower vertical position in Fig. 1. In addition to the library samples, the bottom row of each plate contains pure elemental standards. Both the electrochemical and optical experiments described above produced a 1-d dataset for each sample, which was archived for future analysis and interpretation.

The catalytic current as a function of overpotential was determined via interpretation of the electrochemical voltage sweep data. The catalytic current was calculated for each sample by subtracting an automatically recognized linear residual current from the measured current, as shown in Fig. 2. We note that this analysis protocol is susceptible to overestimation of the catalytic current due to the possibility of electro-oxidation of the sample. An example of sample oxidation is shown in Fig. 2, but the magnitude of the current is negligible compared to the catalytic currents measured at higher overpotentials. Through extensive electrochemical characterization of similar (Fe$\mathrm{Co}-\mathrm{Ni}-\mathrm{Ti}) \mathrm{O}_{\mathrm{x}}$ libraries, we have confirmed that this sample oxidation is typically reversible and thus we assume that corrosion currents are also negligible. In general, additional catalyst stability studies must be performed to validate the relevance of the catalysts for implementation in a solar fuels device. That is, the methods described in this manuscript can be used to identify initial "hit" catalysts, the most promising of which can be pursued with follow-on studies such as long term electrochemical stability. We also note that the calculated current density is with respect to the geometric area and not the

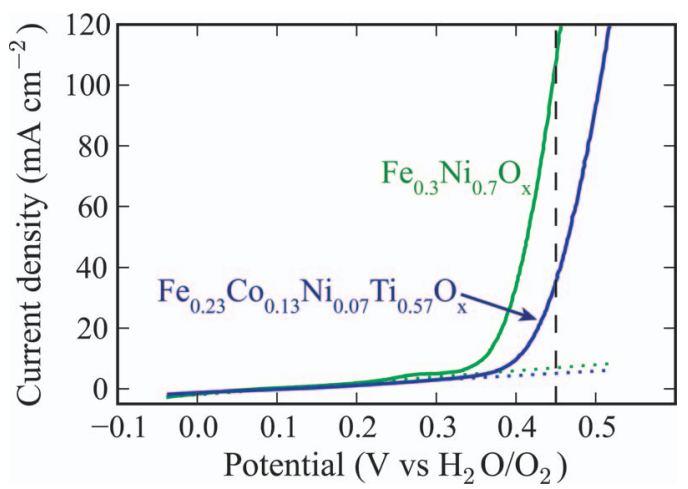

Figure 2. Results from the electrochemical scanning voltage measurement are shown for two samples, each from a composition region of high OER catalytic activity. For each anodic voltage sweep, a linear residual current (dotted line) is automatically identified, from which the OER catalytic current is measured. The catalytic current at $450 \mathrm{mV}$ overpotential (dashed line) is used to compare activity across the composition library. 


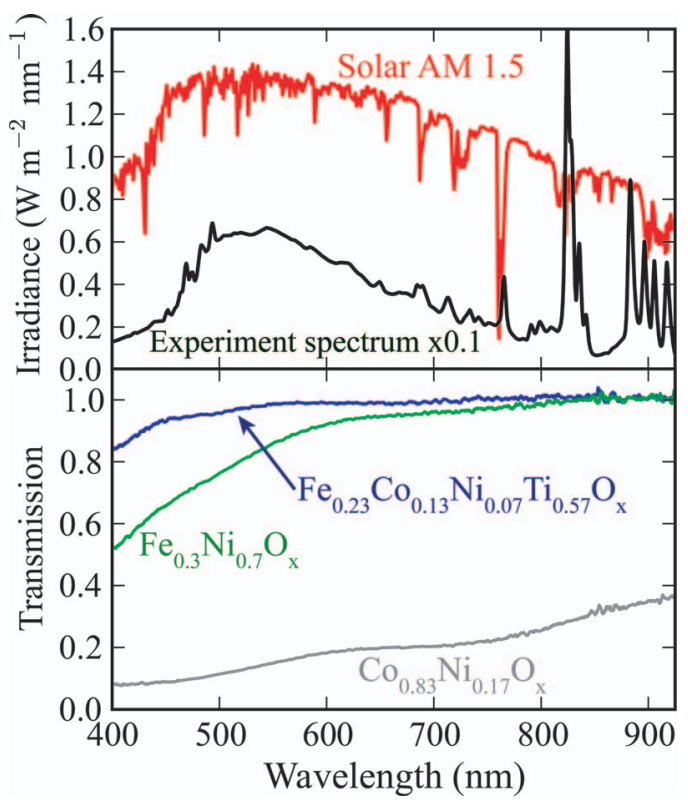

Figure 3. (top) The AM1.5 solar irradiance and the optical experiment irradiance are shown over the wavelength range used for characterization of catalyst transparency. (bottom) Transmission spectra for the catalysts of Fig. 2 are shown along with the spectrum for the least transparent catalyst.

catalytically active surface area. The porous samples are believed to have appreciable specific area compared with the $1 \mathrm{~mm}^{2}$ footprint, but the characterizations of this area and of the porous structure of the films are beyond the scope of the present manuscript.

Each sample's spectral transmission was used to calculate the transmission efficiency of the AM1.5 solar irradiance using Eq. 2. The irradiance used in the experiments is shown along with the AM1.5 spectrum in Fig. 3, which also contains transmission spectra from select samples. In addition to the mesostructure imposed by the structure directing agent, the samples were visibly rough with non-uniform thickness. Every sample appeared to have similar roughness, but the compositional uniformity of the sample morphology is under further investigation.

The FTO coated glass has near perfect transmission over this entire wavelength range and the spectrometer has a flat, low-intensity dark noise spectrum. The processing function of Eq. 5 thus provides a high fidelity transmission spectrum for each sample, which could be used for modeling each sample's optical bandgap. This type of information is important for development of not only photoabsorbers but also photocatalysts, which perform both optical absorption and catalytic functions. While these functional materials are of great interest to JCAP, the focus of the present manuscript is the evaluation of socalled "dark" catalysts.

Given these electrochemical and optical characterizations for each sample, the catalytic efficiency $\eta_{\mathrm{C}, O E R}$ and overall efficiency $\eta_{\mathrm{C}}$ can be readily evaluated given the photoabsorber parameters of Eq. 3. The choice of these parameters strongly dictates the resulting calculated efficiencies and composition trends, which highlights the importance of archiving the electrochemical and optical data so that catalysts can be vetted for new photoabsorbers. For the present purposes, we choose an overpotential of $450 \mathrm{mV}$ vs. $\mathrm{O}_{2} / \mathrm{H}_{2} \mathrm{O}$ and a photocurrent density of $23 \mathrm{~mA} \mathrm{~cm}^{-2}$ under AM1.5 irradiation, which is optimal for water-splitting reactions under certain assumptions. ${ }^{17}$ These conditions correspond to Eq. 3 parameter choices of $V_{\mathrm{PA}, O E R}=450 \mathrm{mV}$ and $J_{\mathrm{PA}, \max }=23 \mathrm{~mA} \mathrm{~cm}^{-2}$.

Performance maps in composition space.- The figures of merit for catalysis, $J_{\mathrm{C}}(450 \mathrm{mV})$, and those described by Eqs. 2 and 4 were analyzed for each library sample. The metal composition of each sample was taken to be that of the precursor printing design. The composition fidelity of the precursor printing has been verified for similar libraries with identical drying and calcination treatments (data not shown). Such studies have also demonstrated the efficient removal of the precursor ligands and block copolymers during the calcination. The oxygen stoichiometry of the samples was not measured, but the compositional map of the catalyst performance is evaluated using the metal stoichiometry, as shown in Fig. 4.

The quaternary composition map of OER current shows nonmonotonic trends with respect to each of the elements. The optimal composition region extends to the $\mathrm{Fe}-\mathrm{Ni}$ binary line with $J_{\mathrm{C}}(450 \mathrm{mV})$ in excess of $70 \mathrm{~mA} \mathrm{~cm}{ }^{-2}$ from $\mathrm{Fe}_{0.5} \mathrm{Ni}_{0.5} \mathrm{O}_{\mathrm{x}}$ to $\mathrm{Fe}_{0.1} \mathrm{Ni}_{0.9} \mathrm{O}_{\mathrm{x}}$. This result is in strong agreement with previous reports of catalysis in this pseudo-binary system. ${ }^{18}$ The catalytic current map also shows a local maximum near the Fe-Co-Ti plane. This composition region exhibits improved catalysis compared to the pure metal oxides, and the active region is bounded by $\mathrm{Ti}$ concentrations of 30 and 70 at.\%. The existence of a finite optimal $\mathrm{Ti}$ concentration is interesting given the marked inactivity of $\mathrm{TiO}_{\mathrm{x}}$.

The compositional variation of $\eta_{C, T}$ is relatively straightforward, and the most significant trends in the optical transmission are the decrease with respect to Co concentration and increase with respect to Ti concentration. The visible appearance of the pure element oxides are translucent red $\mathrm{FeO}_{\mathrm{x}}$, black $\mathrm{CoO}_{\mathrm{x}}$, transparent-white $\mathrm{NiO}_{\mathrm{x}}$, and transparent $\mathrm{TiO}_{x}$, and the measured variation in optical transparency is consistent with a monotonic interpolation of these end-member properties.

The two composition regions noted above, which are locally maximal in the measured catalytic current, are also locally maximal in the map of $\eta_{\mathrm{C}}$. However, the globally maximum composition region for catalytic current is not the globally maximum region in the map of $\eta_{\text {C. }}$ Figs. 2 and 3 contain data for a representative sample from each of these regions $\left(\mathrm{Fe}_{0.23} \mathrm{Co}_{0.13} \mathrm{Ni}_{0.07} \mathrm{Ti}_{0.57} \mathrm{O}_{\mathrm{x}}\right.$ and $\left.\mathrm{Fe}_{0.3} \mathrm{Ni}_{0.7} \mathrm{O}_{\mathrm{x}}\right)$. Fig. 2 shows that both samples provide the target $23 \mathrm{~mA} \mathrm{~cm}^{-2}$ at $450 \mathrm{mV}$ and thus in these regions, $\eta_{C}$ is determined by the transmission efficiency. The transmission spectra in Fig. 3 show that the $\mathrm{Fe}_{0.3} \mathrm{Ni}_{0.7} \mathrm{O}_{\mathrm{x}}$ sample is less transparent in the UV, most likely due to absorption at wavelengths below an optical gap. Due to the very high transparency of the catalysts with composition near $\mathrm{Fe}_{0.23} \mathrm{Co}_{0.13} \mathrm{Ni}_{0.07} \mathrm{Ti}_{0.57} \mathrm{O}_{\mathrm{x}}$, this region exhibits the highest $\eta_{C}$ values in this library. That is, by considering both catalytic and optical efficiency, optimal catalysts are discovered in this surprising composition region. We note that this result is particular to catalysts prepared in this format, and that the excessive catalytic current in the $\mathrm{Fe}_{0.3} \mathrm{Ni}_{0.7} \mathrm{O}_{\mathrm{x}}$ region suggests that the target current may be attained with thinner (or lower areal density) catalysts. Such catalysts could compete with the $\mathrm{Fe}_{0.23} \mathrm{Co}_{0.13} \mathrm{Ni}_{0.07} \mathrm{Ti}_{0.57} \mathrm{O}_{\mathrm{x}}$ catalysts for optimal performance, but an increase in $\eta_{C}$ upon thinning the catalyst is not guaranteed as the resultant material properties cannot be ascertained from the data at hand. For example, the transparency of thinned $\mathrm{Fe}_{0.3} \mathrm{Ni}_{0.7} \mathrm{O}_{\mathrm{x}}$ may remain unchanged if this catalyst suffers from reflective losses. In general such proposed catalysts must be synthesized and screened by the methods described above.

In this study, a mesoporous OER catalyst structure was chosen due to its relevance for incorporation into a solar fuels device. Porous films with thickness on the order of $100 \mathrm{~nm}$ have a significant technological advantage over dense films due to their increased catalytic surface area, which relaxes the requirement on catalyst turnover frequency. To evaluate materials with this technologically relevant format, we constructed performance metrics that conform to the requirements of an operational solar fuels device. As shown in Figs. 3-4, films with this structure can be highly transparent and yield high catalyst efficiency, and the noted composition regions should be further explored for solar fuels applications. New OER catalysts must still be developed for further optimization of solar fuel generation, especially with respect to the required OER overpotential.

We note that the performance metrics described in this manuscript are extrinsic material properties, as opposed to the intrinsic properties typically mapped in a combinatorial materials study (for example, geometric current instead of specific current density). In the discovery and optimization of materials for a specific application, the most 

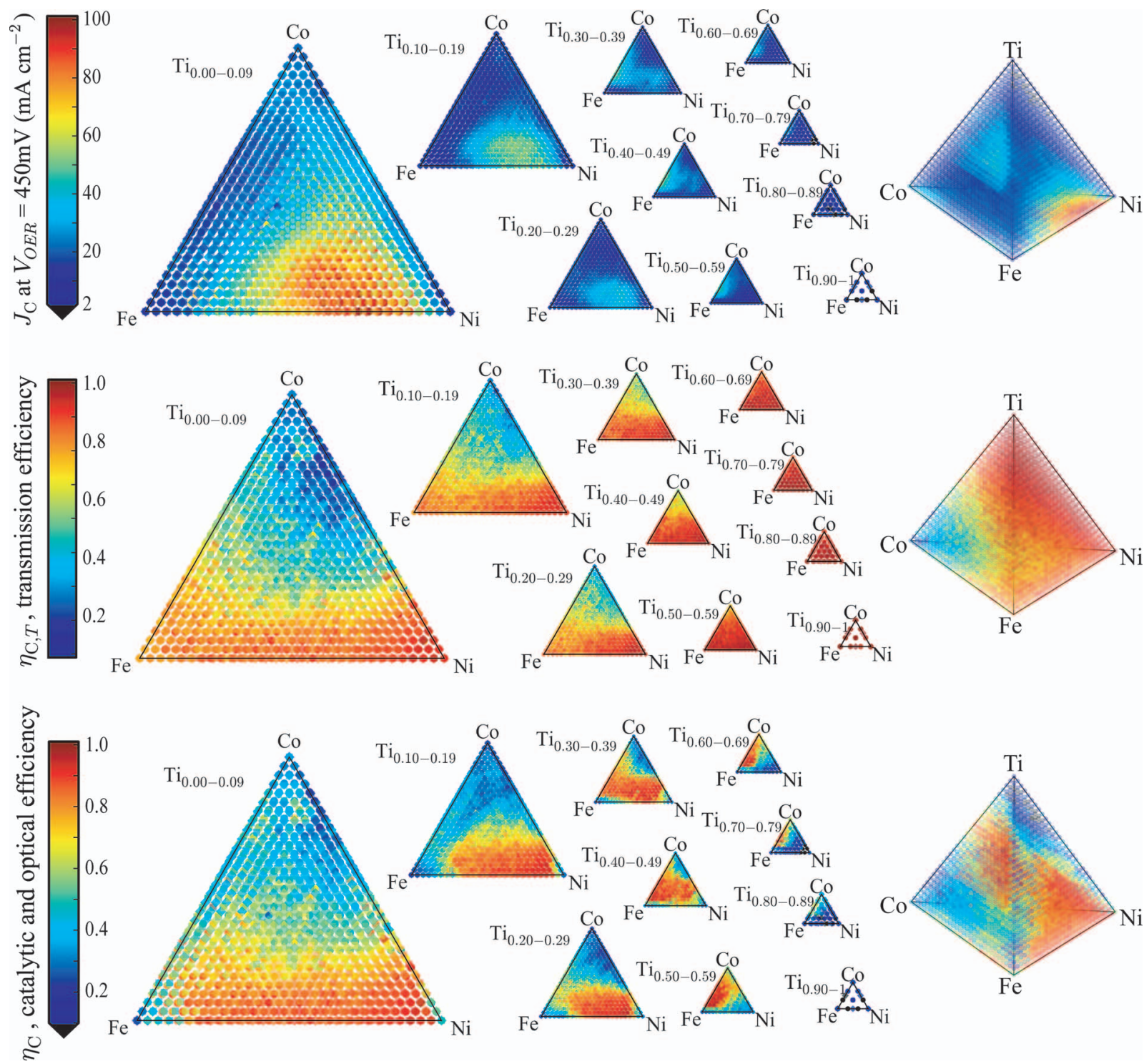

Figure 4. Performance as a function of library composition is shown for the OER catalytic current (top), transparency efficiency (middle) and overall catalyst efficiency (bottom). Each performance metric is mapped onto a stacked-ternary (middle) and tetrahedron (right) representation of the quaternary composition space using a false color scale (left). The tetrahedron plot contains horizontal planes of data points corresponding to 3.3 at. $\%$ intervals of Ti concentration. Sets of 3 planes are plotted together in each of 10 ternary composition plots in the stacked-ternary representation.

pertinent figures of merit will inherently be extrinsic properties of a material in a particular format. We note that the experimental techniques described above can also be used for mapping intrinsic properties, which is essential for evaluating turnover frequency, studying catalyst mechanism and other science endeavors. However, for the purpose of evaluating catalysts for direct incorporation into an operational device, the extrinsic figures of merit described above are most relevant. Employing these application-specific performance metrics bridges the gap between very low throughput experimental evaluation of catalysts in an operating device and traditional combinatorial studies of intrinsic material properties. Using this approach, we have implemented very high throughput methods for screening libraries of solar fuels catalysts.

\section{Conclusion}

To evaluate OER catalysts for solar fuel applications, a catalyst efficiency is derived using parameters of the photoabsorber to which the catalyst would be coupled in an operating device. The efficiency includes OER catalytic current as well as the catalyst's transmission of the solar irradiance. This expression for efficiency is applicable to dark catalysts, which must transmit the solar energy to be converted by the photoabsorber. To enable high-throughput mapping of this catalyst efficiency, both electrochemical and optical screenings are performed on a quaternary metal oxide library at a throughput of $5.5 \mathrm{~s}$ and $3 \mathrm{~s}$ per sample, respectively. Of the 5456 samples in the (Fe-Co-Ni-Ti) $\mathrm{O}_{\mathrm{x}}$ library, two composition regions of interest are identified. The optimal OER catalytic activity is observed near $\mathrm{Fe}_{0.3} \mathrm{Ni}_{0.7} \mathrm{O}_{\mathrm{x}}$, and the optimal catalyst efficiency is observed near $\mathrm{Fe}_{0.23} \mathrm{Co}_{0.13} \mathrm{Ni}_{0.07} \mathrm{Ti}_{0.57} \mathrm{O}_{\mathrm{x}}$. The identification of optimal performance in this composition region highlights the utility of high-throughput experimentation for the discovery of new materials in unexpected composition spaces. The appreciable $\mathrm{Ti}$ concentration in this optimal region prompts future characterization of these catalysts and exploration of related composition spaces. Such discovery from high-throughput searches is crucial for the identification of new materials to enable scalable solar fuel technology.

\section{Acknowledgments}

This material is based upon work performed by the Joint Center for Artificial Photosynthesis, a DOE Energy Innovation Hub, as 
follows: The experiments and data interpretation were supported through the Office of Science of the U.S. Department of Energy under Award No. DE-SC0004993; J.F. acknowledges financial support from the National Science Foundation of China (21003106 and 20873122). The authors thank Lung-Sheng Lin for assistance in fabrication of the cell and William Fisher of Lawrence Berkeley National Laboratory for assistance in fabrication of the capillary for the reference electrode. The authors also thank Dr. Joel Haber, Eric McFarland, Nathan Lewis, Carl Koval and Joachim Lewerenz for helpful discussions.

\section{References}

1. Q. X. Dai, H. Y. Xiao, W. S. Li, Y. Q. Na, and X. P. Zhou, J. Comb. Chem., 7, 539 (2005).

2. N. S. Lewis, Science, 315, 798 (2007).

3. M. G. Walter, E. L. Warren, J. R. McKone, S. W. Boettcher, Q. X. Mi, E. A. Santori, and N. S. Lewis, Chem. Rev., 111, 5815 (2011).
4. J. B. Gerken, J. Y. C. Chen, R. C. Masse, A. B. Powell, and S. S. Stahl, Angew. Chem. Int. Edit., 51, 6676 (2012).

5. M. F. Weber and M. J. Dignam, J. Electrochem. Soc., 131, 1258 (1984).

6. M. Gratzel, Nature, 414, 338 (2001).

7. O. Khaselev and J. A. Turner, Science, 280, 425 (1998).

8. A. J. Sathrum and C. P. Kubiak, J. Phys. Chem. Lett., 2, 2372 (2011).

9. M. Yagi and M. Kaneko, Chem. Rev., 101, 21 (2001).

10. M. Yagi, A. Syouji, S. Yamada, M. Komi, H. Yamazaki, and S. Tajima, Photoch. Photobio. Sci., 8, 139 (2009).

11. E. E. Benson, C. P. Kubiak, A. J. Sathrum, and J. M. Smieja, Chem. Soc. Rev., 38, 89 (2009).

12. T. F. Jaramillo, S. H. Baeck, A. Kleiman-Shwarsctein, K. S. Choi, G. D. Stucky, and E. W. McFarland, J. Comb. Chem., 7, 264 (2005).

13. M. Woodhouse and B. A. Parkinson, Chem. Soc. Rev., 38, 197 (2009).

14. J. E. Katz, T. R. Gingrich, E. A. Santori, and N. S. Lewis, Energ. Environ. Sci, 2, 103 (2009).

15. X. Liu, Y. Shen, R. Yang, S. Zou, X. Ji, L. Shi, Y. Zhang, D. Liu, L. Xiao, X. Zheng, S. Li, J. Fan, and G. D. Stucky, Nano letters, 12, 5733 (2012).

16. J. M. Gregoire, C. Xiang, X. Liu, M. Marcin, and J. Jin, Rev. Sci. Instrum., accepted (2013).

17. J. R. Bolton, S. J. Strickler, and J. S. Connolly, Nature, 316, 495 (1985).

18. D. A. Corrigan, J. Electrochem. Soc., 134, 377 (1987). 